\title{
The fluid structures for soft-sphere potentials via the zero-separation theorems on molecular distribution functions
}

Cite as: J. Chem. Phys. 104, 8058 (1996); https://doi.org/10.1063/1.471522

Submitted: 22 January 1996 . Accepted: 20 February 1996 . Published Online: 31 August 1998

Lloyd L. Lee, Dhananjay Chonasgi, and Enrique Lomba

\section{ARTICLES YOU MAY BE INTERESTED IN}

An accurate integral equation theory for hard spheres: Role of the zero-separation theorems in the closure relation

The Journal of Chemical Physics 103, 9388 (1995); https://doi.org/10.1063/1.469998

A test particle approach to the zero separation theorems of molecular distribution functions

The Journal of Chemical Physics 91, 477 (1989); https://doi.org/10.1063/1.457483

Integral equation theory for uncharged liquids: The Lennard-Jones fluid and the bridge function

The Journal of Chemical Physics 103, 2625 (1995); https://doi.org/10.1063/1.470724
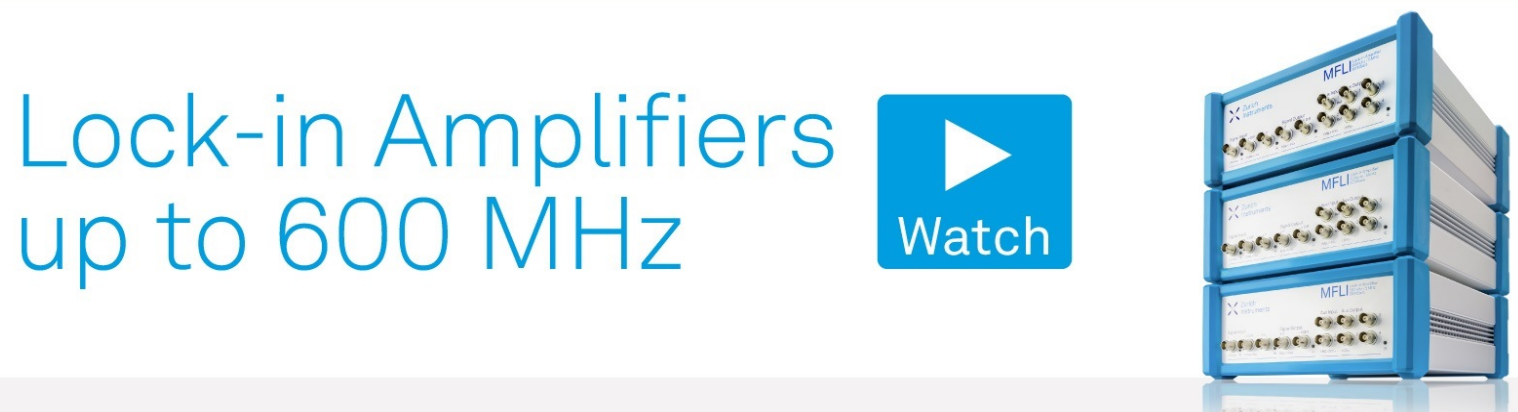

J. Chem. Phys. 104, 8058 (1996); https://doi.org/10.1063/1.471522 


\title{
The fluid structures for soft-sphere potentials via the zero-separation theorems on molecular distribution functions
}

\author{
Lloyd L. Lee and Dhananjay Ghonasgi \\ School of Chemical Engineering and Materials Science, University of Oklahoma, Norman, Oklahoma 73019 \\ Enrique Lomba \\ Instituto de Quimica Fisica Rocasolano CSIC, el Serrano 119, 28006 Madrid, Spain
}

(Received 22 January 1996; accepted 20 February 1996)

\begin{abstract}
We present a class of closures specifically designed to satisfy the zero-separation theorems for the correlation functions $y(r)$ (the cavity function), $\gamma(r)=h(r)-C(r)$ (the indirect correlation), and $B(r)$ (the bridge function) at coincidence $r=0$ for soft-sphere pair potentials. The rationale is to ensure the correct behavior of these correlation functions inside the core $r<\sigma$. Since the coincidence theorems implicate the thermodynamic properties of the bulk fluid: the isothermal compressibility, the internal energy and the chemical potentials, we can hopefully enforce consistency between the structure and thermodynamic properties. We solve the Ornstein-Zernike equation for the Lennard-Jones molecules where plentiful Monte Carlo data are available for testing. It turns out that not only consistency is achieved, we also obtain accurate structures: the pair correlation function $g(r)$, the cavity function, and the bridge function for wide ranges of fluid states $\left(0.72<T^{*}<1.5\right.$, $\left.\rho^{*}<0.9\right)$. Comparison with MC data attests to the accuracy. The closure of the zero-separation type (ZSEP), is sufficiently robust and flexible to ensure not only fulfillment of the zero-separation theorems but also pressure consistency. Success with the Lennard-Jones potential implies its applicability to other similar soft-sphere potentials. () 1996 American Institute of Physics. [S0021-9606(96)50720-8]
\end{abstract}

\section{INTRODUCTION}

Recent trends in the study of fluid structures have strengthened the needs for obtaining the molecular distribution functions from the integral equation (IE) methods. ${ }^{1}$ However, the existing schemes for calculating these structures, with some bright spots here and there, have been weakened by inaccuracies at high densities and low temperatures and the inability to treat more complicated but immensely interesting molecules. The computer simulation methods: Monte Carlo (MC) and molecular dynamics (MD) have supplanted the IE methods in many interesting cases (e.g., the water molecules, adsorption in pores, and polymer melts). Empirical and theoretically based equations of state (or free energy equations: e.g., SAFT ${ }^{2}$ ) can achieve substantial accuracy with faster computer speed. Some important advances in integral equations will have to be made in order for IE to remain viable and competitive.

We have essayed a number of approaches earlier ${ }^{3}$ for simple molecules such as the hard spheres. ${ }^{4,5}$ Currently, we are examining soft sphere molecules. Most common integral equations are based on one of two theoretical formalisms: (i) the Born-Bogoliubov-Green-Kirkwood-Yvon ${ }^{6}$ (BBGKY) hierarchy and (ii) the Ornstein-Zernike (OZ) equation. ${ }^{7}$ Both give the molecular distribution functions, such as the pair correlation function (pcf) $g(r)$. We shall study the OZ approach below:

$$
\gamma(r) \equiv h(r)-C(r)=\rho \int d \mathbf{r}^{\prime} h\left(r^{\prime}\right) C\left(\left|\mathbf{r}-\mathbf{r}^{\prime}\right|\right),
$$

where $h(r)$ is the total correlation function (tcf) $h(r) \equiv g(r)$ $-1 ; C(r)$, the direct correlation function (dcf); $\gamma(r)$, the indirect correlation function (icf); $\rho$ the number density. This equation can be considered as a definition for the dcf $C(r)$. To solve Eq. (1.1), one needs to supplement by a second condition, the closure relation, ${ }^{1}$ which relates the correlation functions to the pair potential $u(r)$. The closure relation can be given formally as

$$
\ln y(r) \equiv \ln g(r)+\beta u(r)=h(r)-C(r)+B(r) .
$$

This equation introduces a new function $B(r)$ the bridge function [where $y(r) \equiv g(r) \exp (\beta u(r))$ is the cavity function, and $\beta \equiv 1 / k T$ is the reciprocal temperature, $k=$ Boltzmann constant]. One of the advantages of the $\mathrm{OZ}$ approach is that the cluster diagrams for the correlation functions are known. For example, the cluster diagram for $B(r)$ is well known. ${ }^{8}$ The bridge function is actually the key to success of the IE method: accurate $B(r)$ will give accurate pcf's, vice versa. However, to calculate "exactly" the bridge function according to the cluster integrals is computationally impossible even for a simple fluid such as hard spheres. Only few low density clusters are known thereof. Approximate IEs [e.g., Percus-Yevick (PY) ${ }^{9}$ and hypernetted chain $(\mathrm{HNC})^{10}$ equations] make estimates of $B(r)$, thus producing approximate pcf's. HNC simply sets $B(r)=0$ identically. PY postulates

$$
B_{\mathrm{PY}}(r) \approx \ln [1+\gamma(r)]-\gamma(r) .
$$


The errors of the approximations show up at high fluid densities and low temperatures. ${ }^{11}$ First, there is the question of thermodynamic consistency. The pcf's from these approximate IEs, when substituted into the thermodynamic formulas for energy $U$, pressure $P$ and isothermal compressibility $K_{T}$, do not yield consistent values (for example, not obeying the Maxwell relations). Thus thermodynamic inconsistencies are telltale indications of the inaccuracies in the approximate closures. Second, these approximate correlation functions fail to match exact machine results from MC and MD.

We have two issues confronting us: one fundamental, and the other practical. (a) The fundamental question is how to obtain the bridge functions accurately from first principles. (b) The practical question is how to construct bridge functions that give accurate and consistent thermodynamic properties. These two questions are, of course, interrelated. Exact correlation functions necessarily give consistent thermodynamic properties (the necessity argument). But consistent thermodynamic properties do not imply "exactness" of the correlation functions (the sufficiency argument). Item $a$ is a far more challenging enterprise, solution of which is tantamount to a solution of the $N$-body problem. In fact, we already know the cluster diagrams of the bridge function. ${ }^{8}$ In addition, its functional expansion in infinite series is also known. ${ }^{12}$ Both are difficult if not impossible to evaluate numerically. Item $b$ is less theoretically based, but more doable and useful. In this article, we shall pursue avenue $b$.

Fundamentally, given a pair potential $u(r)$ in the Hamiltonian, there corresponds uniquely a pcf $g(r)$ for the state of the fluid (preferably away from the phase boundaries). Then all other correlation functions are also uniquely determined through physics. We express this relation between any two correlation functions by a functional: e.g., $B=\phi[\ln y(r)]$, or $B=\psi[\gamma(r)]$. Namely, given $y(r)$ or some related function $\gamma(r)$, one can in principle go through a functional $\psi$ to get $B(r)$. Functionals differ from functions by the former being dependent on all the $r$ values of the argument function $\gamma(r)$ instead of on a single value of $\gamma$. Only under special circumstances will a functional reduce to a function, $B=\hat{B}\left(\gamma^{*}\right)$. We call this the "reducibility" condition. All approximate closures in literature were attempts to achieve this reducibility [e.g., PY, HNC, Martynov-Sarkisov (MS), ${ }^{13}$ BallonePastore-Galli-Gazillo ${ }^{14}$ (BPGG), and modified Verlet $\left.(\mathrm{VM})^{15}\right]$. Some succeeded for particular cases, most failed to achieve consistency. First, we inquire whether, for general pair potentials, such reduction exists (the mathematical existence question). Second, if the functional is reducible (under conditions to be found), is the reduction unique (the uniqueness question). Namely, are there several reductions that will equally satisfy the representation? When both conditions (existence and uniqueness) are met, we say this reduction has achieved unique reducibility, or unique functionality. From pioneering studies by Zerah-Hansen, ${ }^{16}$ Vompe-Martynov, ${ }^{17}$ Llano-Restrepo-Chapman ${ }^{18}$ and Duh-Haymet, ${ }^{19}$ it has become clear that the reducibility for the closures is closely hinged upon (i) the renormalization of the base function $\gamma^{*}=\gamma+\Delta \gamma$, and (ii) optimization of the pair potential: $u(r)=u^{\mathrm{SR}}(r)+u^{\mathrm{LR}}(r)$. If the functional is ever re- ducible, $B$ should be a function of a renormalized function $\gamma^{*}$ for an optimized potential $u^{\mathrm{LR}}$. Thus the question can now be framed mathematically: by searching through the entire function space, can we find (the existence of) a function $\hat{B}\left(\gamma^{*}\right)$ matching the given functional $B=\psi[\gamma(r)]$ by continually adjusting (tuning) the argument function $\gamma^{*}$ through certain combined optimization renormalization? This task is beyond our reach at the present time. Instead, we opt for $b$, by checking the thermodynamic and other consistency conditions for the closures.

There are several aspects of thermodynamic consistency. There is partial consistency (e.g., the pressure consistency ${ }^{20}$ ). And there are the Helmholtz free energy ${ }^{8,21}$ generating functionals: approximate ones (e.g., the HNC generating functional $^{21}$ ) as well as the exact one (a formal expression in terms of coupling parameter integration ${ }^{21}$ ). These generating functionals have the property that their variational derivatives give the closure relation, the $\mathrm{OZ}$ relation and definitions of these correlation functions. The prominent examples are the HNC functional and the RISM functional. ${ }^{22}$ The exact Helmholtz functional suffers again from intractability. Evaluation of such functionals is equivalent to thermodynamic integration. But the integrand is not "exact." New efficient methods in the integration of functionals should be developed first. Approximate functionals give again inconsistent thermodynamic properties (e.g., $P^{\nu} \neq P^{c}$ in HNC). The approach we choose here is to select some boundary conditions and properties (hopefully the right ones) we know that all "exact" correlation functions must satisfy, then incorporate them in the closure expressions at the outset, so the resulting correlations and properties will be consistent with these criteria. These criteria should include the class of zeroseparation theorems (ZST) on the cavity function $y(0)$, the icf $\gamma(0)$, and the bridge function $B(0)$ for reasons to be explained. We characterize our main thrust being the exploration and use of these zero-separation theorems in a new class of closure relations. However, there is no a priori guarantee that such requirements will produce any better correlation functions or properties. Testing is paramount.

Thermodynamic consistency to us means conforming with classical thermodynamic relations. For example, the Helmholtz free energy $A$ obeys the Gibbs-Helmholtz relation

$$
d(\beta A)=U d \beta-(\beta P) d V, \quad \beta=1 / k T .
$$

In order for $A$ to be an exact differential, the cross partial derivatives should be equal:

$$
\left.\frac{\partial U}{\partial V}\right|_{T}=-\left.\frac{\partial \beta P}{\partial \beta}\right|_{V} .
$$

The energy $U^{\prime}$ and virial pressure $P^{v}$ are obtained from the usual formulas ${ }^{1}$ (prime indicates the configurational properties):

$$
\frac{\beta U^{\prime}}{N}=\frac{\rho}{2} \int d \mathbf{r} \beta u(r) g(r),
$$




$$
\frac{\beta P^{\nu}}{\rho}=1-\frac{\rho}{6 k T} \int d \mathbf{r} r \frac{d u(r)}{d r} g(r) .
$$

This is called the $d U-d P$ consistency by Martynov and Vompe. ${ }^{17,23}$ In addition, the virial pressure $P^{\nu}$ after differentiation be equal to the isothermal compressibility $K_{T}$

$$
\left.\frac{\partial \beta P^{\nu}}{\partial \rho}\right|_{T}=K_{T} \equiv \frac{\partial \beta P^{c}}{\partial \rho}=1-\rho \int d \mathbf{r} C(r) .
$$

This is called the $d P \nu-d P c$ consistency ${ }^{20}$ [consistency between the virial theorem, Eq. (1.7) and the compressibility integral, Eq. (1.8)]. The HNC and PY theories, being approximations, do not satisfy this condition. Lomba and Lee ${ }^{3}$ used the Gibbs-Duhem relation as another example of thermodynamic consistency

$$
\left.\frac{\partial P}{\partial \mu}\right\rfloor_{T}=\rho .
$$

In addition to these thermodynamic consistencies, there are the coincidence values $(r \rightarrow 0)$ of the correlation functions: $y(0), \gamma(0)$, and $B(0)$. These must satisfy the zero-separation theorems (to be presented in Sec. II). The ZSTs are not part of the macroscopic thermodynamics and are independent conditions from the latter. Lately, there have been increased interests in the zero-separation theorems for correlation functions. ${ }^{4,24}$ A notable case is the cavity function $y(0)$ for hard spheres, $\ln y(0)=\beta \mu^{\prime}$ the configurational chemical potential. The closures PY and HNC give poor zero-separation values for $y(0)$ and $B(0)$, thus poor $\beta \mu^{\prime}$.

Martynov and Vompe ${ }^{17}$ have used the $d P \nu-d P c$ and $d U-d P$ thermodynamic consistencies in constructing their closures. Lomba and Lee ${ }^{3}$ used the $d P \nu-d P c$ and GibbsDuhem relation as conditions. The arguments were that $d U-$ $d P$ condition is not "effective" in deciding values of correlation functions in the core region $r<\sigma$ ( $\sigma$ being the Lennard-Jones potential size parameter), because both $U$ and $P$ derive most their contributions from function values at $r>0.8 \sigma$. The Gibbs-Duhem relation is based on the chemical potential. The chemical potential is sensitive to $y(r)$, $B(r)$, and $\gamma(r)$ inside the core, $r<\sigma$. Thus the GibbsDuhem Eq. (1.9) should provide "better" determination of $y$ and $B$ at small $r$ 's. In the present work, the ZSTs serve the same purpose and more directly. We directly require $\gamma, y$, and $B$ at $r=0$ to conform to the ZSTs.

In Sec. II, we present the closure, ZSEP, that satisfies the three zero separation theorems. In Sec. III, we present the direct chemical potential formula that yields $\beta \mu^{\prime}$ without coupling parameter integration. In Sec. IV, we apply the ZSEP closure to the Lennard-Jones (LJ) potential. Results of calculation for the correlation functions as well as thermodynamic quantities are compared with simulation data. Section $\mathrm{V}$ draws some conclusions and anticipates future researches.

\section{THE ZERO-SEPARATION THEOREM-BASED CLOSURES}

The coincidence values of the correlation functions $y(0)$, $\gamma(0)$, and $B(0)$ have been known for sometime. ${ }^{25}$ For general potentials, the cavity function is the "excess chemical potential," i.e., the energy required to insert a dimeric molecule of bond length $L$ into a bath of monomers over (in excess of) the energies of inserting the two constituent monomers separately $^{26}$ (see Fig. 1):

$$
-\ln y(L)=\beta \mu_{2}^{\prime}(L)-2 \beta \mu_{1}^{\prime},
$$

where the argument $L$ in $\beta \mu_{2}^{\prime}(L)$ indicates a dimer with bond length $L$. As $L \rightarrow 0$, the dimer merges into a twice strong monomer (a dimer with the same "size" $\sigma$ of a monomer, but with doubled potential energy, $2 \epsilon$ ). Thus

$$
-\ln y(0)=\beta \mu_{2}^{\prime}(0)-2 \beta \mu_{1}^{\prime} .
$$

For hard spheres, the potential energy is either $\infty$ or 0 . Doubling of $\infty$ or 0 gives back $\infty$ or 0 , respectively: $\beta \mu_{2}^{\prime}(0)$ $=\beta \mu_{1}^{\prime}$. Thus we recover $\ln y_{\mathrm{HS}}(0)=\beta \mu_{1}^{\prime}$, the well-known zero-separation theorem for hard spheres. ${ }^{27}$ Equation (2.2) is more general and is applicable to soft-sphere potentials.

The values of $\beta \mu_{1}^{\prime}$ can be obtained (i) from the solution of the integral equation and the direct chemical potential formula (3.1), (ii) from an accurate equation of state (EOS), or (iii) from computer simulation. As will be seen, we shall use the EOSs [route (ii)]. Some evaluation by Monte Carlo method will also be carried out. The value of the twicestrong monomer $\beta \mu_{2}^{\prime}(0)$ can also be obtained via one of the three routes outlined above. Again, we choose route (ii) (EOS) in this study. For other options, we shall discuss in Sec. V.

For the icf, it is known that it obeys the $\mathrm{OZ}$ relation at all $r$, including $r=0 .{ }^{28}$ Thus

$$
\gamma(0)=\frac{\partial \beta P}{\partial \rho}-1-2 \frac{\beta U^{\prime}}{N}+\rho \int d \mathbf{r} g(r)[C(r)+\beta u(r)] .
$$

The integral in (2.3) would be zero in the soft MSA approximation. ${ }^{16}$ Thus we define

$$
I_{\mathrm{MSA}} \equiv \rho \int d \mathbf{r} g(r)[C(r)+\beta u(r)] .
$$

This quantity turns out to be a relatively small number for dense fluids. Its importance shows up only for dilute states.

The bridge function at $r=0$ can be obtain from (1.2), $B(0)=\ln y(0)-\gamma(0)$ :

$$
B(0)=2 \beta \mu_{1}^{\prime}-\beta \mu_{2}^{\prime}-\left[\beta \frac{\partial P}{\partial \rho}-1-2 \frac{\beta U^{\prime}}{N}(4)+I_{\mathrm{MSA}}\right] .
$$

$$
\text { (1) (2) (3) (4) }
$$

It is seen that all terms [except $I_{\mathrm{MSA}}$, term (5)] on the RHS are thermodynamic in nature. The zero-separation theorems serve as the nexus connecting the thermodynamic properties of the bulk fluid with the correlation functions at coincidence! As mentioned before, we have many choices for determining these thermodynamic properties in terms (1) to (4): from the IE itself, or from an equation of state for the bulk fluid, or from simulation. For the sake of testing the zero-separation methodology, we opt in this work using "ex- 
ternally" supplied thermodynamic properties, namely, from the Nicolas equation ${ }^{29}$ of state for Lennard-Jones (LJ) fluids. Other accurate EOSs are also available. ${ }^{30}$

The closure that is flexible enough for this task and has been tested earlier, for example for hard spheres, is the ZSEP closure $^{4}$

$$
B\left(\gamma^{*}\right)=-\frac{\zeta \gamma^{* 2}}{2}\left(1-\frac{\phi \alpha \gamma^{*}}{\left(1+\alpha \gamma^{*}\right)}\right),
$$

where $\gamma^{*}$ is a "renormalized" icf (see below); $\alpha, \phi$, and $\zeta$ are parameters that are to be determined by satisfying the zero-separation theorem Eq. (2.5). Since there are three parameters $(\alpha, \phi$, and $\zeta)$ we need three conditions for their determination. The second condition chosen is the $d P \nu-$ $d P c$ consistency

$$
\left.K_{T}=\beta \frac{\partial P^{\nu}}{\partial \rho}\right]_{T}
$$

The third condition is to require that the energy from ZSEP be the same as (or very close to) the MD energy values of Johnson $^{31}$ (at the same temperature and density of the bulk fluid)

$$
U^{\prime \mathrm{ZSEP}}=U^{\prime \mathrm{MD}} .
$$

We note that (2.8) is not a consistency condition. It does not require any differential equality. It is to enforce the absolute values of energy (same as the MD data). The choice of the criteria appears, at the outset, arbitrary. For example, in Lomba and Lee, ${ }^{3}$ the criteria used were the pressure consistency and the Gibbs-Duhem relation. Vompe and Martynov ${ }^{17}$ used the $d P \nu-d P c$ and $d U-d P$ criteria. As a consequence, the quality of results will depend very much on whether a "judicious" choice was made. The testing of a " "pudding," so to speak, is in the "eating." As we intend to incorporate the zero-separation theorems in the closures, testing will follow to see if this is a good choice.

Another important ingredient for the success of the closure is the use of a renormalized indirect correlation function. Renormalization of $\gamma$ is almost indispensible in achieving the unique functionality requirement, as shown in previous closures proposed by Zerah-Hansen, ${ }^{16}$ Duh-Haymet, ${ }^{19,32}$ Llano-Restrepo and Chapman, ${ }^{18}$ and Vompe-Martynov. ${ }^{17}$ As a trial case, we renormalize using the attractive part of the pair potential. The Lennard-Jones potential is split according to Weeks-Chandler-Andersen ${ }^{33}$ as

$$
u_{\mathrm{LJ}}(r)=4 \epsilon\left[\left(\frac{\sigma}{r}\right)^{12}-\left(\frac{\sigma}{r}\right)^{6}\right]=u_{0}(r)+u_{a}(r),
$$

where

$$
\begin{aligned}
u_{a}(r) & =-\epsilon, \quad r<^{6} \sqrt{2} \sigma \\
& =u_{\mathrm{LJ}}(r), \quad r \geqslant \sqrt[6]{2} \sigma .
\end{aligned}
$$

Thus

$$
\gamma^{*}(r) \equiv \gamma(r)-\beta u_{a}(r)
$$

( $\epsilon$ is the energy parameter, and $\sigma$ is the size parameter for the LJ potential). In literature, more complicated choices have been proposed and tried (Duh-Haymet, ${ }^{19,32}$ Kang and $\left.\mathrm{Ree}^{34}\right)$. The recipes tried were quite varied and with varying degrees of sophistication. Equation (2.11) is not optimized. We use (2.11) due to its simplicity and ease of comparison with previous works where $(2.11)$ was also used. ${ }^{16-18}$

Equations (2.5), (2.7), and (2.8) provide the three conditions needed to uniquely determine the three parameters $\alpha$, $\phi$, and $\zeta$. We shall allow $\alpha, \phi$, and $\zeta$ to be functions of temperature and density of the bulk fluid. Note that Eq. (2.6) reduces to the VM closure if we set $\zeta=1$, and $\phi=1$. Thus (2.6) can be considered as a more versatile version of the VM closure. ZSEP was successful for hard spheres. ${ }^{4}$ It behooves us to investigate whether ZSEP is also effective for soft spheres.

\section{THE DIRECT CHEMICAL POTENTIAL FORMULA}

For the chemical potential, a direct formula is available ${ }^{35}$ that gives the $\beta \mu^{\prime}$ from the correlation functions at the given state without coupling parameter $(\lambda)$ or density integration. The proviso is that the bridge $B$ must be expressed as a unique function (unique functionality) of some known correlation function (renormalized function, if necessary). The formula we present this time is slightly different from Ref. 35:

$$
\begin{aligned}
\beta \mu^{\prime}= & \rho \int d \mathbf{r}[\ln y(r)-h(r) \\
& +(1 / 2) h(r) \gamma(r)+h(r) B(r)]-S^{*},
\end{aligned}
$$

where the star series $S^{*}$ is given by

$$
S^{*}=\rho \int d \mathbf{r} h(r) \int_{0}^{1} d \lambda B(r ; \lambda) .
$$

If $B$ is a unique function of a renormalized icf $\gamma^{*}$, $B=\hat{B}\left(\gamma^{*}\right)$, and

$$
\gamma^{*}(\lambda)=\gamma_{0}(r)+\lambda \gamma(r)
$$

then the $S^{*}$ integral in (3.2) can be evaluated as

$$
S^{*}=\rho \int d \mathbf{r} \frac{h(r)}{\gamma(r)}\left[\int_{x=\gamma^{*}(0)}^{x=\gamma^{*}(1)} d x \hat{B}(x)\right] .
$$

In the present case,

$$
\gamma^{*}(0)=\gamma_{0}(r)=-\beta u_{a}(r), \quad \text { and } \gamma^{*}(1)=\gamma(r)-\beta \mu_{a}(r) \text {. }
$$

Thus Eq. (3.4) can be integrated accordingly with $\hat{B}$ given by (2.6). The analytical expression is given below in indefinite form:

$$
\begin{aligned}
\int_{0}^{x} d x \hat{B}(x)= & -\frac{\zeta(\alpha x)^{3}}{6 \alpha^{3}}+\frac{\zeta \phi}{6 \alpha^{3}}\left[(1+\alpha x)^{3}-\frac{9}{2}(1+\alpha x)^{2}\right. \\
& \left.+9(1+\alpha x)-3 \ln (1+\alpha x)-\frac{11}{2}\right]
\end{aligned}
$$


TABLE I. The parameters $\alpha, \phi$, and $\zeta$ used in the ZSEP closure (2.6) that satisfy the zero-separation theorems: Eqs. (2.5), (2.7), and (2.8).

\begin{tabular}{lllll}
\hline \hline$T^{*}$ & $\rho^{*}$ & \multicolumn{1}{c}{$\alpha$} & \multicolumn{1}{c}{$\phi$} & \multicolumn{1}{c}{$\zeta$} \\
\hline 1.5 & 0.4 & 0.90 & 1.0 & 0.5356 \\
1.5 & 0.6 & 0.70 & 1.022 & 0.80 \\
1.5 & 0.7 & 0.69 & 1.015 & 0.90 \\
1.5 & 0.8 & 0.745 & 1.004 & 0.98 \\
1.5 & 0.9 & 0.825 & 0.999 & 1.05 \\
1.0 & 0.8 & 0.725 & 1.005 & 0.99 \\
0.81 & 0.8 & 0.70 & 1.0065 & 1.0 \\
0.72 & 0.85 & 0.65 & 1.005 & 1.0 \\
\hline \hline
\end{tabular}

This formula will be used in the evaluation of the chemical potentials from correlation functions obtain from the ZSEP closure.

\section{RESULTS OF CALCULATION}

We propose to study a typical soft-sphere potential: the Lennard-Jones potential. All units will be expressed in terms of the LJ parameters: thus temperature $T^{*}=k T / \epsilon$, and density $\rho^{*}=\rho \sigma^{3}$. Closure (2.6) has been solved together with the OZ equation (1.1). Numerical solution of the integral equation was carried out with 1024 grid points in $r$ and grid size $\Delta r=0.02 \sigma$. Convergence was achieved when the absolute convergence of the Cauchy sequence of $\gamma$ became inferior to an $\delta_{\max }<0.2 E-04$. More stringent criteria have been used with little difference in the answer. The densities studied were for conditions where simulation data are available: for $T^{*}=1.5, \quad \rho^{*}=0.4,0.6,0.7,0.8$, and $0.9 ;$ for $T^{*}=1.0$, $\rho^{*}=0.8$; for $T^{*}=0.81, \rho^{*}=0.8$; and for $T^{*}=0.72, \rho^{*}=0.85$. The parameters $\alpha, \phi$, and $\zeta$ determined are listed in Table I.

With these parameters, we can obtain the structures $B(r), y(r), C(r)$, and $g(r)$ and the thermodynamic properties accurately. The validity extends over ranges of density as high as $\sim 0.9$ and temperatures as low as 0.72 . In addition, the thermodynamic values are pressure consistent because of the requirement (2.7): the virial pressure be equal to the compressibility pressure.

The zero separation values of the bridge function $B_{\text {ZSEP }}(0)$ at eight state conditions are given in Table II, plus

TABLE II. The coincidence values of the bridge function $B(0)$ of ZSEP for the Lennard-Jones potential.

\begin{tabular}{llcccc}
\hline \hline & & \multicolumn{4}{c}{$B(0)$} \\
\cline { 3 - 6 }$T^{\mathrm{a}}$ & $\rho^{\mathrm{a}}$ & $\mathrm{MC}^{\mathrm{a}}$ & $\mathrm{EOS}^{\mathrm{b}}$ & $\mathrm{ZSEP}^{\mathrm{c}}$ & $\mathrm{PY}$ \\
\hline \multirow{2}{*}{1.5} & 0.4 & -0.93 & -1.04 & -1.0 & -2.1 \\
& 0.6 & -3.88 & -3.87 & -3.82 & -5.7 \\
& 0.7 & -7.03 & -7.19 & -7.04 & -9.29 \\
& 0.8 & -12.46 & -12.7 & -12.36 & -15.1 \\
& 0.9 & -20.45 & -21.49 & -20.36 & -24.23 \\
1.0 & 0.8 & -15.18 & -15.55 & -15.24 & -17.0 \\
0.81 & 0.8 & -17.28 & -16.74 & -17.07 & -17.97 \\
0.72 & 0.85 & -25.74 & -24.52 & -25.8 & -23.27 \\
\hline \hline
\end{tabular}

${ }^{a} \mathrm{MC}$ from Llano-Restrepo and Chapman (Ref. 18).

${ }^{\mathrm{b}} \mathrm{EOS}$ from Nicolas et al. (Ref. 29).

'ZSEP from Eq. (2.6).

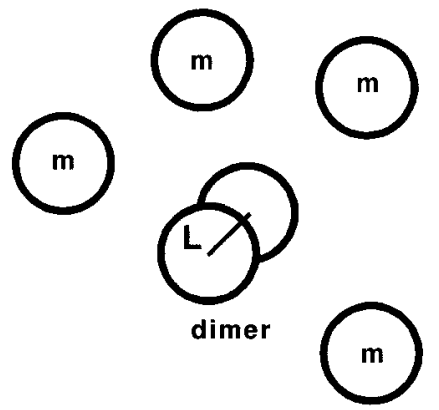

FIG. 1. A dimer molecule at infinite dilution in a bath of monomers $(m)$. The dimer is formed of two monomers fused to a bond length of $L$. In the limit $L \rightarrow 0$, we form a coincident dimer or a strong "monomer" with same size $(\sigma)$ but twice energetic $(2 \epsilon)$.

the MC, EOS, and PY values. For most states, ZSEP is within $0.1 \%-4 \%$ of the MC numbers. The EOS values are listed for the purpose of verification, since condition (2.5) enforces equality between ZSEP and EOS. Let us explain how these EOS thermodynamic properties were obtained. Terms 1, 3, and 4 [Eq. (2.5)] for $\beta \mu_{1}^{\prime}, \beta \partial / P \partial \rho$, and $U^{\prime}$ were straightforward calculations from the Nicolas equation for LJ. Term 2, the coincident dimer $\beta \mu_{2}^{\prime}$ was calculated from the Nicolas EOS for binary mixtures of LJ molecules, assuming van der Waals one-fluid (vdW1) mixing rules, with the coincident dimer at infinite dilution in the monomers (see Fig. 1). This is an approximation, since we are not all that certain about the vdW1 theory for the strong monomer (coincident dimer) in solution. To ascertain the validity, we have carried out MC simulations at $T^{*}=1.5$ for several densities to test $\beta \mu_{2}^{\prime}$. The results are presented in Table III, together with the MC monomer $\beta \mu_{1}^{\prime}$ values (see Fig. 2). Canonical ensemble $(N-V-T)$ was used. $108 \mathrm{LJ}$ molecules were arranged initially on a fcc lattice and subsequently "melted" in 10 million random walk moves (these were discarded). Afterwards for every 1000 moves, 1000 fictitious particle insertions ${ }^{36}$ were attempted. These included separate insertions of a LJ monomer as well as a coincident dimer (with twice the monomer $\epsilon$ ) into a bath of 108 monomers. After equilibration, another 10 million configurations were generated. The cutoff distance of the LJ potential was $2.5 \sigma$. Long range corrections were applied to recover the full $\mathrm{LJ}$ properties. For the infinitely dilute dimer, the long-range correction was twice the magnitude of the monomer correction. Statistics (error bars) were obtained from packet averages after every $1 \times 10^{6}$ insertions.

We note that there was an earlier study on Henry's law constants $^{37}$ for the infinite dilute dimer. Their values check with the present estimation (Fig. 2). The simulation data are filled (darkened) symbols (triangles for $\beta \mu_{1}^{\prime}$ and squares for $\left.\beta \mu_{2}^{\prime}\right)$. The EOS values are solid lines. The chemical potentials $\beta \mu_{2}^{\prime}$ can be back-calculated from the Llano-Restrepo MC data on ln $y(0)$ according to (2.2) and are shown as open rhombics (i.e., from $\beta \mu_{2}^{\prime}=2 \beta \mu_{1 \mathrm{MC}}^{\prime}-\ln y(0)_{\text {Llano-MC }}$ ). We see that the vdW1 $\beta \mu_{2}^{\prime}$ overestimates (less negative than) MC data. There is agreement between the present MC (Table III) calculations and the derived Llano-Restrepo values. The 
TABLE III. The chemical potentials of monomers and the coincident dimer in Lennard-Jones fluid.

\begin{tabular}{|c|c|c|c|c|c|c|c|}
\hline \multirow[b]{2}{*}{$T^{\mathrm{a}}$} & \multirow[b]{2}{*}{$\rho^{\mathrm{a}}$} & \multicolumn{3}{|c|}{$\beta \mu_{1}^{\prime}$} & \multicolumn{3}{|c|}{$\beta \mu_{2}^{\prime}$} \\
\hline & & $\mathrm{MC}^{\mathrm{a}}$ & EOS & $\mathrm{ZSEP}^{\mathrm{b}}$ & $\mathrm{MC}^{\mathrm{a}}$ & EOS & From $\ln y(0)^{\mathrm{c}}$ \\
\hline \multirow[t]{5}{*}{1.5} & 0.4 & $-1.329 \pm 0.0053$ & -1.34 & -1.35 & $-5.362 \pm 0.012$ & -4.9 & -5.24 \\
\hline & 0.6 & $\cdots$ & -1.19 & -1.28 & $\cdots$ & -6.46 & -6.77 \\
\hline & 0.7 & $-0.403 \pm 0.0024$ & -0.485 & -0.8 & $-7.05 \pm 0.058$ & -6.58 & -6.81 \\
\hline & 0.8 & $\ldots$ & 0.88 & 0.2 & $\ldots$ & -5.91 & -6.35 \\
\hline & 0.9 & $3.423 \pm 0.421$ & 3.2 & 2.1 & $-4.62 \pm 0.911$ & -4.11 & -4.16 \\
\hline 1.0 & 0.8 & $\cdots$ & -2.3 & -3.1 & $\cdots$ & -13.3 & -14.3 \\
\hline 0.81 & 0.8 & $\cdots$ & -4.72 & -5.67 & $\cdots$ & -18.8 & -19.7 \\
\hline 0.72 & 0.85 & $\cdots$ & -5.38 & -7.0 & $\cdots$ & -22.2 & -23.3 \\
\hline
\end{tabular}

${ }^{\mathrm{a}} \mathrm{MC}$ values from this work.

${ }^{\mathrm{b}} \mathrm{ZSEP}$ calculated from (2.6) and the direct $\beta \mu_{1}^{\prime}$ formula (3.1).

${ }^{\mathrm{c}} \beta \mu_{2}^{\prime}$ inferred from MC data of Llano-Restrepo: $\beta \mu_{2}^{\prime}=2 \beta \mu_{1}^{\prime}-\ln y(0) . \beta \mu_{1}^{\prime}$ from MC was used when available. Otherwise, $\beta \mu_{1}^{\prime}$ from EOS was substituted.

qualitative trends are also correct. There are some discrepancies in monomer $\beta \mu_{1}^{\prime}$ from EOS too as compared with MC: $\beta \mu_{1_{\mathrm{EOS}}^{\prime}}^{\prime}=3.2$ and $\beta \mu_{1 \mathrm{MC}}^{\prime}=3.4$ at $\rho^{*}=0.9$. The uncertainties in the MC data is \pm 0.4 . The differences are not that substantial. Due to cancellation of errors $\left[B(0)=2 \beta \mu_{1}^{\prime}-\beta \mu_{2}^{\prime}\right.$ $-\gamma(0)]$, the $B_{\mathrm{EOS}}(0)$ values are surprisingly close to the MC values of Llano-Restrepo. ${ }^{18}$ Term 5 in Eq. (2.5) was obtained directly from the IE solution. Any differences between ZSEP and EOS after numerical iterative solution of the OZ are the residual inconsistency. Table II shows that the residuals are quite small (within 5\%). We consider the consistency check to be satisfactory (between EOS and ZSEP). The $B(0)$ values from the PY closure are shown as counter-examples. They are for the most part too negative, from $3 \%$ (the best case) to $100 \%$ off the MC values, especially at higher temperatures $\left(T^{*}=1.5\right)$. We conclude that the PY closure does not satisfy the zero-separation theorem equation (2.5) for the states considered.

For a same separation $r$, Duh and Haymet ${ }^{19}$ proposed plotting the the values $B(r)$ vs $\gamma^{*}(r)$ as check of the closure relations. We construct the Duh-Haymet plot in Fig. 3

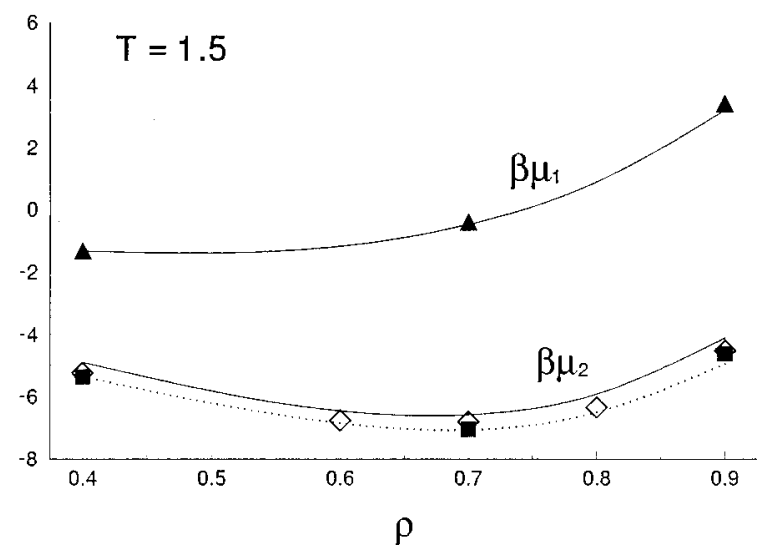

FIG. 2. The chemical potentials of monomer and coincident dimer $(L=0)$ at $T^{*}=1.5$ for the Lennard-Jones potential. Solid lines: Equation of state results [Nicolas (Ref. 29)]. Dotted line: Simulation results from Ghonsagi et al. (Ref. 37). $\mathbf{\Delta}$ : Present MC simulation for monomers. $\mathbf{~}$ : Present MC simulation for coincident dimers (at infinite dilution). $\diamond:$ Derived values from the MC simulation of $\ln y(0)$ of Llano-Restrepo et al. (Ref. 18). for $B\left(\gamma^{*}\right)$ of ZSEP and compare with MC. We observe that the ZSEP results follow the MC simulation data of LlanoRestrepo et al. extremely well, from zero separation (the right extremities of the curves) to contact. The $B$ vs $r$ plots at temperatures: $T^{*}=1.5,1.0,0.81$, and 0.72 are shown in Figs. 4(a) and 4(b). The ZSEP closure equation (2.6) provides equally accurate results.

We observe from Fig. 3 at $\rho^{*}=0.4$ to 0.9 that the five curves are not coincident (there is branching in the $B-\gamma^{*}$ curves). From experience, the degree of conformality in the Duh-Haymet plots is an indication of the success of the renormalization of $\gamma$. (Collapsing of curves means better renormalized $\gamma^{*}$ ). This lack of collimation indicates that the renormalization (2.11) has not quite achieved the unique functionality requirement. More work on renormalization of $\gamma^{*}$ is needed.

The coincidence values of $\gamma(0)$ are shown in Table IV. We observe the close agreement of the $\gamma(0)$ from $\mathrm{MC}^{31}$ and from the ZSEP. Although the EOS values [Eq. (2.3)] were not directly used in enforcing ZSEP, the agreement between EOS and ZSEP is also reasonable. [For ZSEP, Eq. (2.5) was required]. The PY $\gamma(0)$ are too low, especially at lower tem-

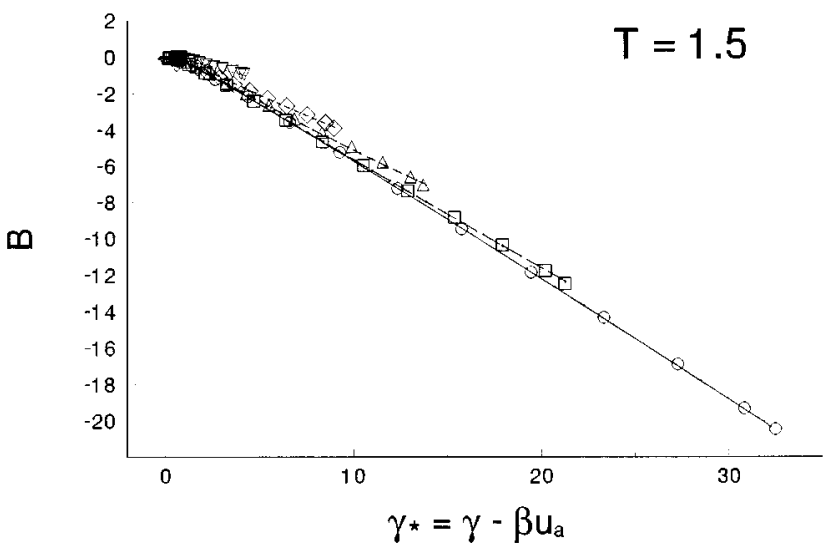

FIG. 3. The ZSEP bridge function $\hat{B}\left(\gamma^{*}\right)$ for Lennard-Jones molecules at $T^{*}=1.5$. Symbols are from the MC data of Llano-Restrepo et al. (Ref. 18). O: $\rho^{*}=0.9 ; \square: \rho^{*}=0.8 ; \triangle: \rho^{*}=0.7 ; \diamond: \rho^{*}=0.6 ; \nabla: \rho^{*}=0.4$. Lines are from ZSEP prediction. Close agreement between ZSEP and MC is achieved. 
(a)

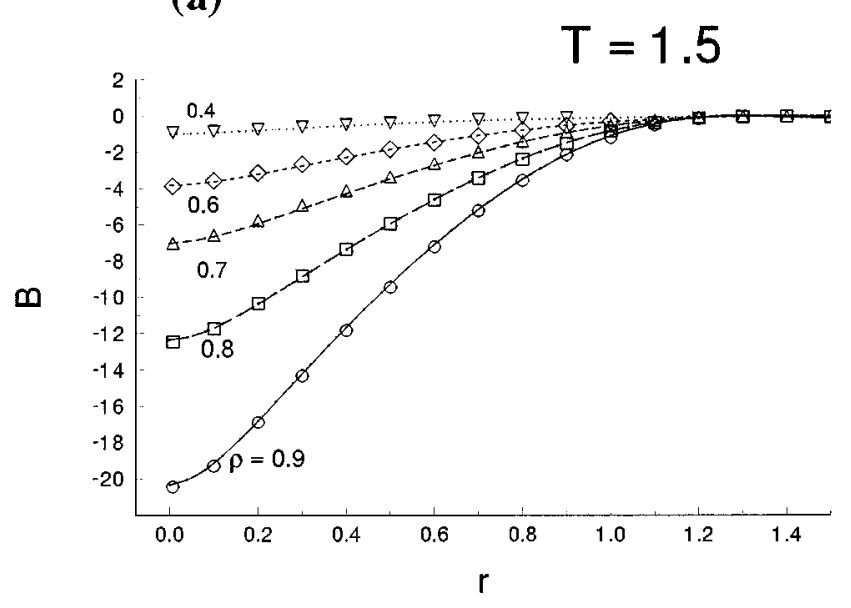

(b)

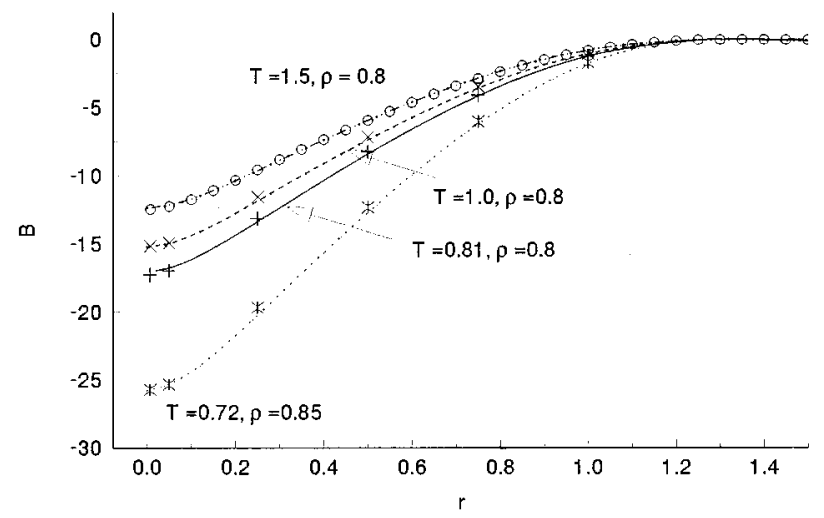

FIG. 4. The bridge function as predicted by the ZSEP closure, Eq. (2.6). Symbols are MC data of Ref. 18. Lines are from ZSEP. (a) For temperature $T^{*}=1.5$. (b) For densities $\rho^{*}=0.8$, and 0.85 . Close agreement is observed for high as well as low densities.

TABLE IV. The coincidence values of the indirect correlation function $\gamma(0)$ for Lennard-Jones fluid.

\begin{tabular}{lcccccc}
\hline \hline & & \multicolumn{5}{c}{$\gamma(0)$} \\
\cline { 3 - 7 }$T^{\mathrm{a}}$ & $\rho^{\mathrm{a}}$ & $\mathrm{MC}^{\mathrm{a}}$ & ZSEP $^{\mathrm{b}}$ & \multicolumn{1}{c}{ PY } & Eq. $(2.3)^{\mathrm{c}}$ & \multicolumn{1}{c}{$I_{\text {MSA }}$} \\
\hline 1.5 & 0.4 & 3.52 & 3.57 & 3.57 & 3.26 & 0.285 \\
1.5 & 0.6 & 8.28 & 8.33 & 7.84 & 7.95 & 0.111 \\
1.5 & 0.7 & 13.02 & 13.18 & 11.80 & 12.80 & -0.319 \\
1.5 & 0.8 & 20.59 & 20.61 & 17.9 & 20.39 & -0.713 \\
1.5 & 0.9 & 31.81 & 31.55 & 27.46 & 32.01 & -1.45 \\
1.0 & 0.8 & 24.86 & 24.85 & 19.94 & 24.3 & -0.758 \\
0.81 & 0.8 & 27.59 & 27.54 & 20.93 & 26.08 & -0.995 \\
0.72 & 0.85 & 38.24 & 38.5 & 26.63 & 36.01 & -1.88 \\
\hline \hline
\end{tabular}

${ }^{\mathrm{a}} \mathrm{MC}$ from Llano-Restrepo and Chapman (Ref. 18).

${ }^{\mathrm{b}}$ Terms in (2.3) are from the Nicolas EOS (Ref. 29). $I_{\mathrm{MSA}}$ from the solution of the integral equation.

'ZSEP from Eq. (2.6).

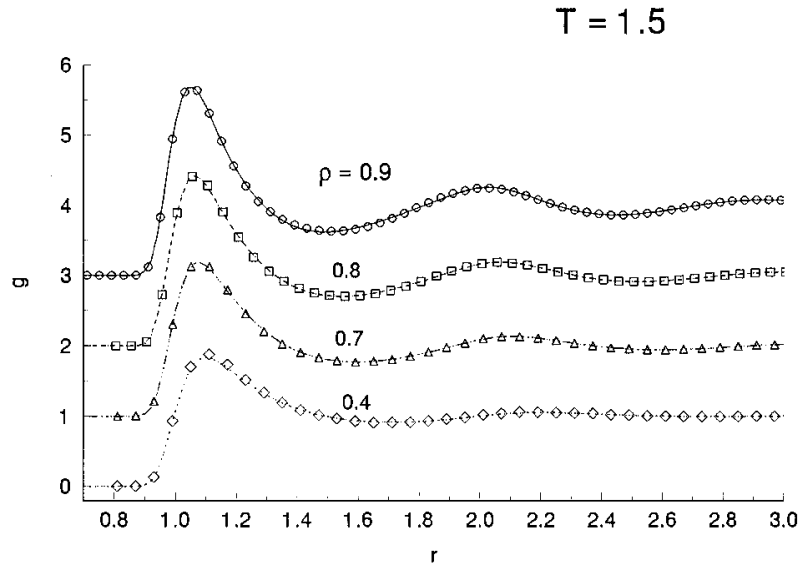

FIG. 5. The pair correlation function as predicted by the ZSEP closure, Eq. (2.6). Symbols are MD data of Johnson (Ref. 31) at $T^{*}=1.5$. Lines are from ZSEP. The curves have been shifted up with a décalage of unity for display purpose. The first peaks and oscillations are in good accord with MC data.

peratures. Consequently, the PY cavity functions are too low.

The pair correlation functions $g(r)$ are compared in Figs. 5 and 6 at two different states $\left(T^{*}=1.5\right.$ or $\rho^{*} \sim 0.8$, 0.85). The MD data (symbols) are from Johnson. ${ }^{31}$ For all the states studied, they are in close agreement, from low densities up to $\rho^{*}=0.9$ and for temperatures down to $T^{*}=0.72$. Low density results are also satisfactory. The first peak heights are well reproduced. The oscillations are in phase. The usual defects of IEs are cured and no longer present a problem.

The cavity functions are compared in Figs. 7 and 8: one set at $T^{*}=1.5$, and the other at $\rho^{*} \sim 0.8$. Again good agreement with MC data is in evidence. The zero-separation values are well reproduced. Next, we compare the thermodynamic properties.

Thermodynamic properties. Figure 9 shows the internal energy $\beta U^{\prime} / N$, the virial pressure $Z=\beta P^{\nu} / \rho$, and the configurational chemical potential $\beta \mu_{1}^{\prime}$. The MD data (symbols) are from Johnson ${ }^{31}$ and the present work. The lines are



FIG. 6. The pair correlation function as predicted by the ZSEP closure, Eq. (2.6). Symbols are MD data of Johnson (Ref. 31). Lines are from ZSEP. Temperatures vary from 0.72 to 1.0 . Densities $=0.8$ and 0.85 . The curves have been shifted up for display. 


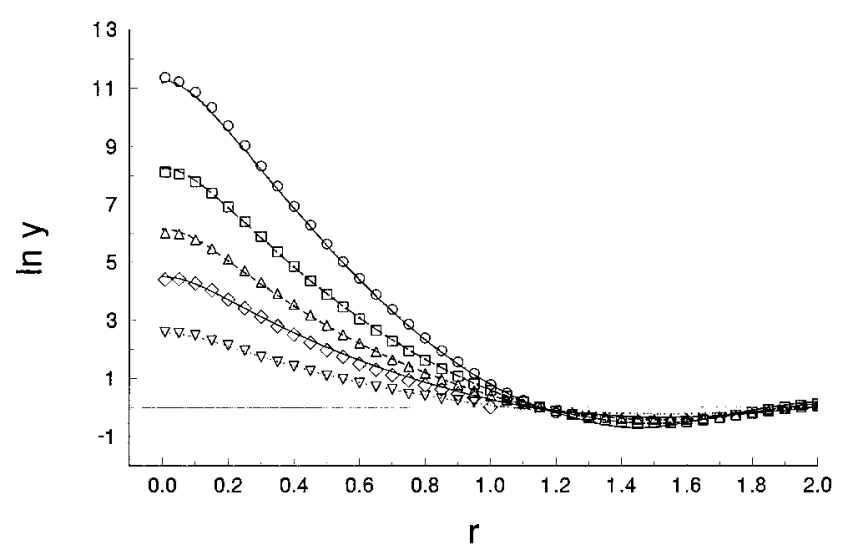

FIG. 7. The cavity function is predicted by the ZSEP closure, Eq. (2.6). $T^{*}=1.5$. Symbols are MC data of Ref. 18. Lines are from ZSEP. From top down: density $\rho^{*}=0.9,0.8,0.7,0.6$, and 0.4 , respectively.

from Nicolas EOS and the present ZSEP calculations. The energy and the pressure are well predicted by ZSEP. The chemical potential from ZSEP is valid up to $\rho^{*} \sim 0.6$. For $\rho^{*}>0.7$, the ZSEP values are lower than the EOS and MC values: i.e., $\mathrm{ZSEP}<\mathrm{EOS}<\mathrm{MC}$. We have mentioned that the renormalization (2.11) was deemed inadequate judging from the Duh-Haymet plots. This affected the chemical potential calculation at high densities, where even small inaccuracies in unique functionality became sensitive and magnified. Equation (2.11) does not affect much the results for the correlation functions $g(r), B(r)$, and $\ln y(r)$. This is attributed to the robustness of the functional form (2.6) and use of the zero-separation theorem (2.5).

Since the internal energy was used as a criterion in determining the ZSEP closure, we compare the $\beta U^{\prime} / N$ in Table V. We have also included the virial pressures for comparison. The numbers from all calculations (MD, EOS, and ZSEP) are in close agreement (mostly within 1\%). This justifies the condition (2.8). The pressure, being a more sensitive quantity than the energy, is surprisingly well predicted.

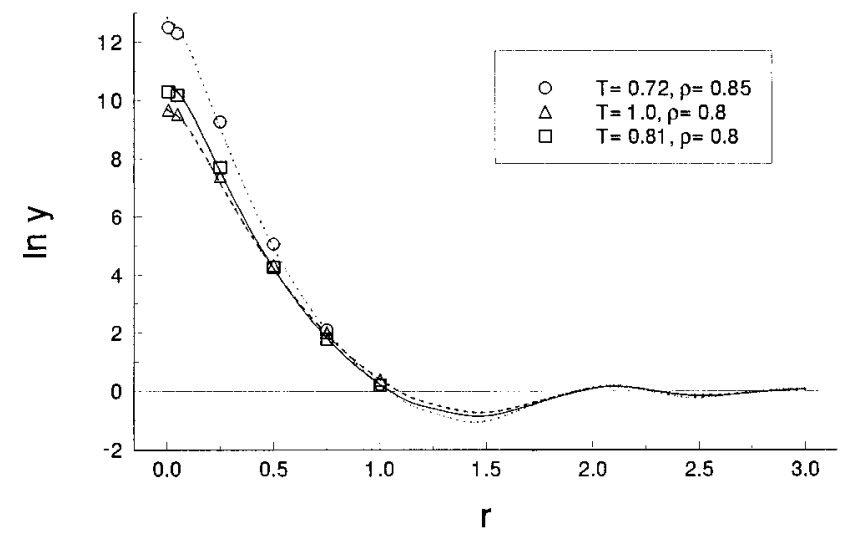

FIG. 8. The cavity function as predicted by the ZSEP closure, Eq. (2.6). $T^{*}=0.72,0.81$, and 1.0. Symbols are MC data of Ref. 18. Lines are from ZSEP. Densities vary from 0.8 to 0.85 .

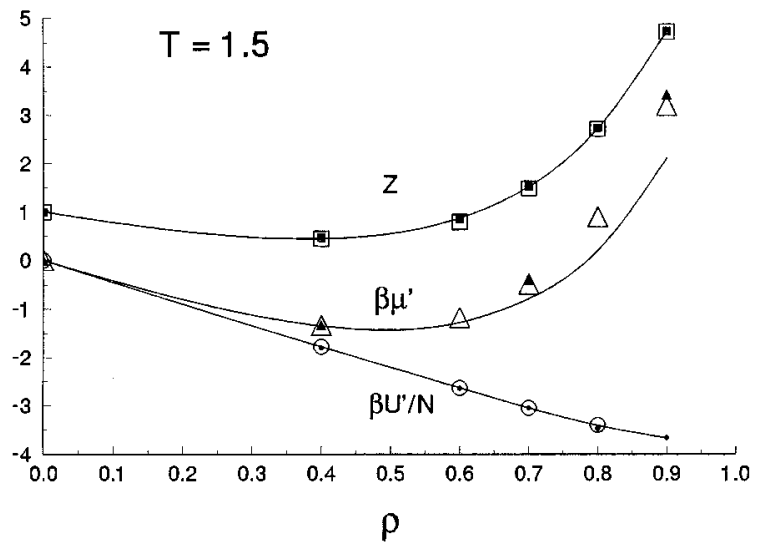

FIG. 9. Thermodynamic properties of the Lennard-Jones fluid at $T^{*}=1.5$. $Z=P^{v} / \rho k T$, the virial compressibility, $\beta \mu^{\prime}=$ excess chemical potential. $\beta U^{\prime} / N=$ excess internal energy. Lines: ZSEP results. Open symbols $(\bigcirc, \triangle, \square)$ : equation of state results (Ref. 29). Filled symbols $(\boldsymbol{\bullet}, \mathbf{\Lambda}, \boldsymbol{\square})$ : MC simulation results.

Figure 10 displays the isothermal compressibility obtained from the direct correlation function, Eq. (1.8). Compared to EOS, ZSEP gives very fiduciary values. Note that due to the pressure consistency, the compressibility pressure $P^{c}$ is equal to the virial pressure $P^{v}$.

\section{CONCLUSIONS}

We have shown that (i) the ZSEP closure (2.6), though containing a dose of empiricism, is sufficiently general in producing accurate bridge functions $B(r)$, cavity functions $y(r)$, and pair correlation functions $g(r)$ for the LennardJones fluid over a wide spectrum of densities and temperatures: $0.72<T^{*}<1.5$ and $\rho^{*}<0.9$. The three conditions: the zero-separation theorem on $B(r)$, the pressure consistency $d P^{v}=d P^{c}$, and the energy requirement are sufficient in determining the closure parameters $\alpha, \phi$, and $\zeta$. The thermodynamic quantities: pressure, isothermal compressibility, internal energy, and chemical potential are all accurately reproduced. (ii) The renormalization of the indirect correlation $\gamma^{*}$ is important in obtaining the chemical potential through the direct formula, and the unique function relation

TABLE V. The internal energy and virial pressure from ZSEP for LennardJones fluids.

\begin{tabular}{|c|c|c|c|c|c|c|c|}
\hline \multirow[b]{2}{*}{$T^{\mathrm{a}}$} & \multirow[b]{2}{*}{$\rho^{\mathrm{a}}$} & \multicolumn{3}{|c|}{$\beta U^{\prime} / N$} & \multicolumn{3}{|c|}{$\beta P_{v} / \rho$} \\
\hline & & $\mathrm{MC}^{\mathrm{a}}$ & $\mathrm{EOS}^{\mathrm{b}}$ & $\mathrm{ZSEP}^{\mathrm{c}}$ & $\mathrm{MC}^{\mathrm{a}}$ & $\mathrm{EOS}^{\mathrm{b}}$ & $\mathrm{ZSEP}^{\mathrm{C}}$ \\
\hline 1.5 & 0.4 & -1.8 & -1.781 & -1.784 & 0.465 & 0.447 & 0.447 \\
\hline 1.5 & 0.6 & -2.639 & -2.635 & -2.629 & 0.856 & 0.791 & 0.868 \\
\hline 1.5 & 0.7 & -3.052 & -3.0458 & -3.0456 & 1.516 & 1.479 & 1.516 \\
\hline 1.5 & 0.8 & -3.466 & -3.4 & -3.414 & 2.737 & 2.715 & 2.723 \\
\hline 1.5 & 0.9 & -3.674 & -3.657 & -3.673 & 4.717 & 4.721 & 4.719 \\
\hline 1.0 & 0.8 & -5.533 & -5.523 & -5.52 & 1.288 & 1.309 & 1.316 \\
\hline 0.81 & 0.8 & -7.046 & -7.027 & -7.026 & 0.106 & 0.204 & 0.179 \\
\hline 0.72 & 0.85 & -8.5 & -8.43 & -8.498 & 0.386 & 0.490 & 0.36 \\
\hline
\end{tabular}

${ }^{\mathrm{a}} \mathrm{MD}$ from Johnson (Ref. 31).

${ }^{b}$ EOS from the Nicolas EOS (Ref. 29).

${ }^{\mathrm{c}}$ ZSEP from Eq. (2.6). 




FIG. 10. Isothermal compressibility, $\beta \partial P / \partial \rho-1$ for Lennard-Jones fluid at $T^{*}=1.5$. Line: equation of state of Nicolas (Ref. 29). $\diamond:$ ZSEP results.

$B-\gamma^{*}$. (i) and (ii) are related, but can also be decoupled to a certain extent. If the closure form is robust, as in (2.6), it can give good correlation functions despite some weaknesses in renormalization. The direct chemical potential formula is sensitive to the degree of unique functionality in the renormalization. Currently, $\beta \mu^{\prime}$ obtained from (3.6) is valid up to $\rho^{*} \leqslant 0.6$. We believe that it can be improved beyond $\rho^{*} \geqslant 0.6$ if a better renormalization of $\gamma^{*}$ is used.

We have demonstrated that it is possible with ZSEP to obtain very accurate correlation functions for the LennardJones fluid. We attribute this primarily to the closure (2.6) and the enforcement of the zero-separation theorems (2.2), (2.3), and (2.5). They ensure the correct behavior of $B(r)$, $\gamma(r)$, and $y(r)$ at $r=0$ inside the core.

To obtain the thermodynamic quantities in the zero separation $B(0)$, Eq. (2.5), we have used an equation of state $\left(\right.$ Nicolas $\left.^{29}\right)$. This choice is for convenience. (If one already knows the properties of Lennard-Jones fluids, there is probably little incentive to go through an integral equation to recalculate the same quantities!) However, the goal of this investigation is to see if the concept of enforcing the zeroseparation theorems works. The outcome from a combination of closure (2.6) and conditions (2.5), (2.7), and (2.8) has been shown to be very promising. The agreement with $\mathrm{MC}$ data is excellent. This avenue of investigation proves productive.

Once the concepts have been proven valid, we propose further studies for the future. Improvements are sought: (i) to obtain the thermodynamic quantities autochthonously (i.e., from the integral equation itself); (ii) to search for better renormalization of the icf $\gamma^{*}$ so as to attain unique functionality. To achieve (i), the chemical potential of the coincident dimer $\beta \mu_{2}^{\prime}$ poses as a problem: it is a heterogeneous species, though spherical, from the monomeric LJ spheres. This value cannot be obtained from solving for the pure LJ fluid alone, no matter what closure is used. We shall devise means to treat this. To achieve (ii), better theoretically based renormalization of $\gamma$ or similar functions is needed. The monomer chemical potential and the Duh-Haymet plots are sensitive measures of unique functionality, and consequently the renormalization scheme. These can be used as guides.
We have chosen the Lennard-Jones potential as the subject of study due to the availability of simulation data. The zero-separation approach, once proven valid, can be generalized to treat other soft-sphere potentials.

\section{ACKNOWLEDGMENTS}

We thank Dr. J. Karl Johnson for generously providing the molecular dynamics data on the Lennard-Jones correlation functions for use in this study. E.L. acknowledges support from the Spanish Dirección General de Investigación Científica y Técnica under Grant No. PB94-0112.

${ }^{1}$ See, e.g., in L. L. Lee, Molecular Thermodynamics of Nonideal Fluids (Butterworths, Boston, 1988).

${ }^{2}$ M. S. Wertheim, J. Stat. Phys. 35, 35 (1984); 42, 459 (1986); 42, 477 (1986); J. Chem. Phys. 85, 2929 (1986); 87, 7323 (1987); W. G. Chapman and K. E. Gubbins, Pure Appl. Chem. 59, 53 (1987); C. G. Joslin, C. G. Gray, W. G. Chapman, and K. E. Gubbins, Mol. Phys. 62, 843 (1987); W. G. Chapman, G. Jackson, and K. E. Gubbins, ibid. 65, 1057 (1988).

${ }^{3}$ E. Lomba and L. L. Lee, Int. J. Thermophys 17, 663 (1996).

${ }^{4}$ L. L. Lee, J. Chem. Phys. 103, 9388 (1995).

${ }^{5}$ E. Lomba, M. Alvarez, L. L. Lee, and N. G. Almarza, J. Chem. Phys. 104, 4180 (1996)

${ }^{6}$ J. Yvon, La Théorie Statistique des Fluides et l'Equation d' Etat (Actualités Scientifiques et Industrielles, No. 203, Paris, 1935).

${ }^{7}$ L. S. Ornstein and F. Zernike, Proc. Akad. Sci. Amsterdam 17, 793 (1914).

${ }^{8}$ T. Morita and K. Hiroike, Progr. Theor. Phys. 23, 1003 (1960).

${ }^{9}$ J. K. Percus and G. J. Yevick, Phys. Rev. 110, 1 (1958).

${ }^{10}$ T. Morita, Progr. Theor. Phys. 20, 920 (1958); J. M. J. van Leeuwen, J. Groeneveld, and J. de Boer, Physica 25, 792 (1959).

${ }^{11}$ D. Levesque, Physica 32, 1985 (1966); J. A. Barker and D. Henderson, Rev. Mod. Phys. 48, 587 (1976).

${ }^{12}$ See, e.g., L. L. Lee, J. Chin. I. Ch. E. 19, 337 (1988).

${ }^{13}$ G. A. Martynov and G. N. Sarkisov, Mol. Phys. 49, 1495 (1983).

${ }^{14}$ P. Ballone, G. Pastore, G. Galli, and D. Gazillo, Mol. Phys. 59, 275 (1986).

${ }^{15}$ L. Verlet, Mol. Phys. 41, 183 (1980); S. Labik, A. Malijevski, and W. R. Smith, ibid. 73, 87 (1991); E. Lomba, C. Martin, M. Lombardero, and J. A. Anta, J. Chem. Phys. 96, 6132 (1992).

${ }^{16}$ J. P. Hansen and G. Zerah, Phys. Lett. 108A, 277 (1985); G. Zerah and J. P. Hansen, J. Chem. Phys. 84, 2336 (1986).

${ }^{17}$ G. A. Martynov and A. G. Vompe, Phys. Rev. E 47, 1012 (1993); A. G. Vompe and G. A. Martynov, J. Chem. Phys. 100, 5249 (1994).

${ }^{18}$ M. Llano-Restrepo and W. G. Chapman, J. Chem. Phys. 97, 2046 (1992); ibid. 100, 5139 (1994).

${ }^{19}$ D.-M. Duh and A. D. J. Haymet, J. Chem. Phys. 97, 7716 (1992).

${ }^{20}$ D. S. Hall and W. R. Conkie, Mol. Phys. 40, 907 (1980).

${ }^{21}$ K. Hiroike, Progr. Theor. Phys. 24, 317 (1960).

${ }^{22}$ D. Chandler and H. C. Andersen, J. Chem. Phys. 57, 1930 (1972).

${ }^{23}$ K. Hiroike, J. Phys. Soc. Japan. 15, 771 (1960).

${ }^{24}$ Y. Zhou and G. Stell, J. Stat. Phys. 52, 1389 (1988).

${ }^{25}$ L. L. Lee and K. S. Shing, J. Chem. Phys. 91, 477 (1989).

${ }^{26}$ T. Boublik, Mol. Phys. 59, 371 (1986).

${ }^{27}$ E. Meeron and A. J. F. Siegert, J. Chem. Phys. 48, 3139 (1968); E. W. Grundke and D. Henderson, Mol. Phys. 24, 269 (1972).

${ }^{28}$ Y. Rosenfeld and N. W. Ashcroft, Phys. Rev. A 20, 1208, 2162 (1979); L. L. Lee, T. H. Chung, and K. E. Starling, Fluid Phase Equil. 12, 105 (1983).

${ }^{29}$ J. J. Nicolas, K. E. Gubbins, W. B. Streett, and D. J. Tildesley, Mol. Phys. 37, 1429 (1979).

${ }^{30}$ J. K. Johnson, J. A. Zollweg, and K. E. Gubbins, Mol. Phys. 78, 591 (1993).

${ }^{31}$ J. K. Johnson, MD data on Lennard-Jones fluids, private communication 1995.

${ }^{32}$ D.-M. Duh and A. D. J. Haymet, J. Chem. Phys. 103, 2625 (1995). 
${ }^{33}$ J. D. Weeks, D. Chandler, and H. C. Andersen, J. Chem. Phys. 54, 5237 (1971).

${ }^{34}$ H. S. Kang and F. H. Ree, J. Chem. Phys. 103, 9370 (1995).

${ }^{35}$ L. L. Lee, J. Chem. Phys. 99 (1992).
${ }^{36}$ B. Widom, J. Chem. Phys. 54, 2808 (1963); J. Phys. Chem. 86, 869 (1982).

${ }^{37}$ D. Ghonasgi, M. Llano-Restrepo, and W. G. Chapman, J. Chem. Phys. 98 , 5662 (1993). 